\title{
Cargas de trabalho de enfermagem em unidade de internação psiquiátrica e a saúde do trabalhador
}

\author{
Nursing workload in a psychiatric inpatient unit and workers' health \\ Cargas de trabajo de enfermería en una unidad de internación psiquiátrica y la salud de los trabajadores
}

\author{
Silvia Regina Carvalho de Souza ${ }^{\text {; }}$ Elias Barbosa de Oliveira ${ }^{\text {II }}$; Maria Yvone Chaves Mauro ${ }^{\text {III }}$; \\ Rosane Mello ${ }^{I V}$; Celia Caldeira Fonseca Kestemberg ${ }^{V}$; Glaudston Silva de PaulaVI
}

\begin{abstract}
RESUMO: Objetivou-se, neste estudo, identificar as cargas de trabalho de enfermagem em uma unidade de internação psiquiátrica e analisar as repercussões para a saúde dos trabalhadores. Método qualitativo, descritivo, tendo como campo uma unidade de internação em um hospital psiquiátrico situado no município do Rio de Janeiro (Brasil). Utilizou-se a técnica de entrevista semiestruturada com 30 trabalhadores de enfermagem em 2014. Aplicada a análise de conteúdo aos depoimentos, chegou-se aos seguintes resultados: as cargas de trabalho física e psíquica acarretam estresse ocupacional e desgastes, como a peculiaridade do cuidado de pacientes nas emergências psiquiátricas, a insuficiência de recursos humanos e materiais e a reduzida autonomia. Conclui-se que cabe à organização investir em ações preventivas, a partir das cargas identificadas pelos trabalhadores, no intuito de promover a saúde do grupo e motivar a participação na tomada de decisões que revertam em melhoria das condições de trabalho.
\end{abstract}

Palavras-Chave: Carga de trabalho; recursos humanos de enfermagem; saúde mental; enfermagem.

\begin{abstract}
This qualitative, descriptive study to identify the nursing workload in a psychiatric inpatient unit and analyze the impact on nursing staff health was conducted at a psychiatric hospital in Rio de Janeiro municipality, Brazil. Semi-structured interviews of 30 nursing workers were conducted in 2014. Content analysis yielded the following results: the physical and psychological workload causes job stress and wear, because of the peculiarities of care for patient in psychiatric emergencies, insufficient human resources, and lack of autonomy. It was concluded that the organization should invest in preventive actions in view of workload identified by workers, so as to promote group health and encourage participation in decision making to improve working conditions.

Keywords: Workload; nursing staff; mental health; nursing.
\end{abstract}

RESUMEN: Estudio cuyo objetivo fue identificar las cargas de trabajo de enfermería en una unidad de internación psiquiátrica y analizar las repercusiones para la salud de los trabajadores. Método cualitativo, descriptivo, que tiene como campo una unidad de internación en un hospital psiquiátrico situado en la ciudad de Rio de Janeiro (Brasil). Se ha utilizado la técnica de entrevista semiestructurada junto a 30 trabajadores de enfermería, en 2014. Aplicado el análisis de contenido a las declaraciones, se llegó a los resultados siguientes: las cargas de trabajo física y psíquicas provocan estrés laboral y desgastes, como la peculiaridad del cuidado de pacientes en las emergencias psiquiátricas, la insuficiencia de recursos materiales y humanos y la poca autonomía. Se concluyó que le cabe a la organización invertir en acciones preventivas a partir de las cargas identificados por los trabajadores con el fin de promover la salud del grupo y estimular la participación en la toma de decisiones que se transformen en mejoría de las condiciones de trabajo.

Palabras Clave: Carga de trabajo; personal de enfermería; salud mental; enfermería.

\section{INTRODUÇÃO}

O presente estudo surgiu a partir da problemática relacionada ao absenteísmo e às licenças para tratamento de saúde de profissionais de enfermagem que trabalhavam em unidade de internação de um grande hospital psiquiátrico, que possui como característica principal o acolhimento de pacientes em crise. Esta modalidade de atendimento em saúde mental, por acolher pacientes na fase aguda da doença, exige do trabalhador permanente estado de alerta e a capacidade de lidar com a imprevisibilidade de quadros clínicos diversos. Deste modo, o estresse físico e mental é acentuado em decorrência das longas jornadas de tra-

\footnotetext{
IEnfermeira. Mestre Enfermagem. Especialista em Enfermagem Psiquiátrica e Saúde Mental. Rio de Janeiro, Brasil. E-mail: silvinhanurse@yahoo.com.br. IIEnfermeiro. Pós-Doutor em Álcool e Drogas. Professor Associado de Enfermagem em Saúde Mental e Psiquiatria (Mestrado) e Graduação da Universidade do Estado do Rio de Janeiro. Brasil. E-mail: eliasbo@oi.com.br.

IIIEnfermeira. Doutora e Mestre em Enfermagem. Professora do Programa de Pós-Graduação da Faculdade de Enfermagem da Universidade do Estado do Rio de Janeiro. Brasil. E-mail: mycmauro@uol.com.br.

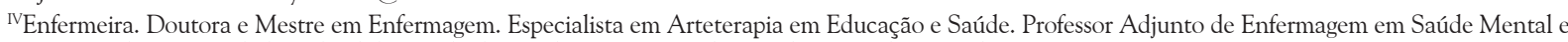
Psiquiatria da Universidade Federal do Estado do Rio de Janeiro. Brasil. E-mail: rosane.dv@gmail.com.

vEnfermeira. Doutora e Mestre em Psicologia Social. Professora Adjunta de Enfermagem em Saúde Mental e Psiquiatria da Universidade do Estado do Rio de Janeiro. Brasil. E-mail: celiaskestenberg@gmail.com.

vi Mestre em Enfermagem. Especialista em Saúde da Família. Professor Assistente, Faculdade Gama e Souza. Rio de Janeiro, Brasil. E-mail: glaudston.silva@gmail.com.
} 
balho e da necessidade de os profissionais intervirem nos episódios de agitação psicomotora dos pacientes devido aos riscos de auto e heteroagressividade.

Fundamentando-se na associação entre queixas e fatores referidos, indica-se que o absenteísmo e/ou licenças para tratamento de saúde dos trabalhadores, por acarretar problemas na dinâmica laboral e conflitos entre os componentes da equipe, têm sido fonte de desgaste de enfermeiros, técnicos e gerência do serviço. Aqueles que permanecem no posto de trabalho se sentem sobrecarregados, considerando que, na maioria das vezes, não há substituição imediata do profissional afastado, resultando que os demais se desdobram para manter a qualidade do atendimento de pacientes, e outras solicitações, profissionais ou não, inerentes ao trabalho em equipes do contexto ${ }^{1}$.

Quanto às condições de trabalho na psiquiatria, os trabalhadores convivem com instalações físicas, espaços pequenos e inadequados para o desenvolvimento das atividades. Há problemas relativos à insuficiência de profissionais, a escassez de materiais e equipamentos, ocasionando a formação de equipes pequenas e sobrecarregadas de trabalho. A precarização da força de trabalho também se faz presente tendo em vista os baixos salários, os vínculos empregatícios terceirizados (precarizados) e com insuficiência de formação continuada em serviço².

Em face das novas diretrizes da política nacional de saúde mental, o trabalho de enfermagem psiquiátrica vem passando por importantes transformações nas últimas décadas e, com isso, novas práticas estão sendo assimiladas pelo profissional, na tentativa de aperfeiçoar a assistência integral ao paciente com transtorno mental. Consequentemente, os profissionais têm sentido a necessidade de refletir sobre suas ações, pois o processo de trabalho é constituído por conflitos e resistências, necessitando ser repensado no cotidiano em que se constrói ${ }^{3}$.

A avaliação dos serviços de saúde mental tem recebido atenção crescente por causa do incentivo da Organização Mundial de Saúde em aumentar a qualidade dos serviços prestados à população. Nesta perspectiva, pacientes, familiares e equipes de profissionais têm sido cada vez mais enfatizados como parte integrante e necessária na avaliação de serviços. No entanto, pacientes e suas famílias têm recebido atenção privilegiada, em detrimento do estudo sobre as repercussões do trabalho sentidas pela equipe de profissionais ${ }^{4}$.

O presente estudo teve como objetivos identificar as cargas de trabalho de enfermagem em uma unidade de internação psiquiátrica e analisar as repercussões para a saúde dos trabalhadores. $\mathrm{O}$ intuito é o de contribuir com pesquisas que coloquem em evidência a nocividade das cargas de trabalho para a saúde mental e física dos trabalhadores de enfermagem e ratificar a relevância de medidas voltadas para a prevenção e a promoção da saúde do grupo.

\section{REVISÃo DA LITERATURA}

No setor saúde, poucas áreas de conhecimento e atuação são tão complexas e com tanta transversalidade de saberes e práticas quanto a psiquiatria. $O$ trabalho neste campo faz com que os profissionais sejam confrontados diariamente com a dor e o sofrimento de pacientes que, por terem doenças crônicas e redicivantes, nutrem grande dependência em relação aos trabalhadores e serviço. Conviver com a precariedade da existência e com a sua fragilidade acarreta grande sofrimento aos trabalhadores que, a todo instante, são lembrados da vulnerabilidade que o sofrimento psíquico produz ${ }^{5}$.

Acrescenta-se que a transição no perfil das características do trabalho em psiquiatria vem sendo acompanhada de sérios problemas de âmbito gerencial. A falta de investimentos em recursos humanos tem se agravado e gerado déficit de profissionais qualificados, achatamento salarial e não reposição de pessoal. Na tentativa de contornar esta problemática, muitas instituições recorrem à contratação de trabalhadores temporários e terceirizados que, além de não solucionar o problema, acarreta uma sobrecarga de trabalho devido à rotatividade e tendo como consequência altos níveis de estresse, sintomas depressivos e ansiosos no grupo ${ }^{6}$.

Deste modo, os trabalhadores de enfermagem são submetidos a cargas de trabalho que representam o conjunto de esforços despendidos pelo trabalhador para atender às exigências da tarefa, incluindo esforços físicos, cognitivos e os psicoafetivos, que agem de forma integrada. A carga quantitativa (demasiado trabalho, atenção concentrada e contínua pressão de tempo e outros) diz respeito ao volume de trabalho mental exigido dentro de determinada unidade de tempo. A carga qualitativa refere-se ao nível de complexidade da atividade e à possibilidade de aplicação, concomitantemente com interesses significativos, experiências, capacidades e potenciais do trabalhador?

$\mathrm{Na}$ enfermagem, estudos apontam que as cargas de trabalho estão associadas às condições ergonômicas do ambiente laboral, acarretando prejuízos à saúde em decorrência de lesões osteomusculares quando se tratam de situações muito prolongadas, intensas ou frequentes. Ainda, percebeu-se nos estudos o significativo aumento dos custos decorrentes do absenteísmo e dos acidentes de trabalho, sendo enfatizada a importância de se conhecer os recursos que os trabalhadores possuem para o enfrentamento, principalmente da carga psíquica, causadora dos desgastes físicos e emocionais da equipe de enfermagem e de saúde ${ }^{8}$.

\section{Metodologia}

Estudo qualitativo de caráter descritivo considerando que o objeto investigado pertence a uma realidade ligada ao mundo do trabalho com significados, motivos, 
aspirações, crenças, valores e atitudes resultantes de ação humana, apreendidos por meio do cotidiano, da vivência e da explicação das pessoas que vivenciam determinada situação 9 . O projeto foi aprovado pelo Comitê de Ética em Pesquisa e protocolado no Comitê Nacional de Ética em Pesquisa (CAAE) com o n ${ }^{\circ}$ 23356414.6.0000.5259.

Em atendimento à Resolução n ${ }^{\circ} 466 / 12$, após a assinatura do termo de consentimento livre e esclarecido, participaram do estudo 30 trabalhadores de enfermagem de uma unidade de internação, de um hospital psiquiátrico, de grande porte, situado no Município do Rio de Janeiro. Os critérios adotados na inclusão dos participantes foram ser do quadro permanente da instituição ou contratado para trabalhar na psiquiatria e atuar há, pelo menos, seis meses no campo de estudo, sendo excluídos os trabalhadores com menos de seis meses de atuação naquele serviço e os que estivessem de férias ou outros tipos de afastamento.

Informou-se que a participação dos depoentes seria voluntária e que teriam o direito de se retirarem da pesquisa em qualquer fase. Garantiu-se o sigilo dos depoimentos e ratificou-se que os resultados seriam somente apresentados em eventos e publicados em revistas científicas. Na transcrição dos depoimentos, foram adotadas as seguintes convenções: enfermeiro (letra E) e técnico de enfermagem (TE), seguidos de um número, de acordo com a ordem de entrada no texto.

A coleta de da dos ocorreu no segundo semestre de 2014, no próprio local de trabalho, após a seleção e o convite. Utilizou-se a técnica de entrevista semiestruturada, que combinou perguntas fechadas e abertas com a possibilidade de o indivíduo discorrer sobre o tema em questão, favorecendo a contextualização de experiências, vivências e sentidos ${ }^{9}$. $\mathrm{Na}$ obtenção dos dados, foi utilizado um instrumento para o registro das características sociodemográficas e profissionais dos sujeitos, seguido de um roteiro contendo questões relacionadas ao ambiente, às condições de trabalho e às cargas, cujas respostas foram gravadas em meio digital.

Realizada a transcrição, os depoimentos foram submetidos à técnica de análise de conteúdo - um conjunto de técnicas analíticas das comunicações, visando obter, por procedimentos sistemáticos e objetivos de descrição dos significados das mensagens, indicadores (quantitativos ou não) que permitam a inferência de conhecimentos relativos às condições de produção/recepção (variáveis inferidas) destas mensagens ${ }^{10}$. Utilizando os critérios de representatividade, homogeneidade, reclassificação e agregação dos elementos do conjunto, chegou-se às seguintes categorias: Imprevisibilidade e vigilância: cargas psíquicas; Condições inadequadas de trabalho; A reduzida autonomia frente à organização do trabalho; e Implicações das cargas de trabalho para a saúde: o sofrimento no trabalho.

\section{Resultados e Discussão}

\section{Características dos participantes do estudo}

Foram investigados 6(20\%) enfermeiros e 24(80\%) técnicos de enfermagem, sendo 25(83,4\%) do sexo feminino e $5(16,6 \%)$, do sexo masculino. Quanto ao estado civil, 15(50\%) eram casados, 7(23,3\%), solteiros, 6(20\%), divorciados e 2(6,6\%), viúvos. A faixa etária variou de 26 a 60 anos, a maioria - 20(66,6\%) - com idades de 35 a 55 anos. Quanto ao número de vínculos empregatícios, 15(50\%) possuíam um vínculo e $15(50 \%)$ dois vínculos, cumprindo carga horária de 30 horas semanais - 22(73,3\%) e 8(26,7\%) mais de 50 horas, incluindo os dois vínculos. No que diz respeito à jornada de trabalho, 24(80\%) trabalhavam em regime de turnos diurno e/ou noturno (plantão) de 12 por 60 horas e os demais, distribuídos nos períodos da manhã e tarde.

O trabalho por turnos ou noturno é caracterizado como uma atividade realizada em horário não usual, que inclui turnos alternantes ou fixos, com escalas bem variadas. Diante disso, surge a necessidade de uma atenção especial aos efeitos cumulativos, originados por fatores que provocam inegáveis prejuízos ao trabalhador, associados a problemas de saúde física e psicológica, mudanças biológicas, comportamentais e dificuldades sociais. Os trabalhos por turno e noturno passaram a ser incluídos no grupo V da CID-10: Transtornos Mentais e do Comportamento relacionados com o trabalho, considerado como má adaptação à organização do horário de trabalho, para o desenvolvimento de transtorno do ciclo vigília-sono devido a fatores não orgânicos ${ }^{11}$.

Apesar de grande parte dos trabalhadores se encontrar na fase de vida produtiva, um fator agravante do desgaste é o gênero, pois a maioria é do sexo feminino, casada e acumula mais de um vínculo empregatício. Por desenvolverem uma terceira jornada de trabalho relacionada às atividades do lar, há maior desgaste psicofísico, principalmente ao se considerar a carga horária semanal total de trabalho. Tal realidade de trabalho, além de aumentar o cansaço físico e mental, também expõe o trabalhador a riscos de erros por fadiga e acidentes no trabalho?

\section{Imprevisibilidade e vigilância: cargas psíquicas}

Apesar de a Reforma Psiquiátrica ter em seu bojo a proposta de trabalho multi e interdisciplinar, ainda cabe à enfermagem a realização de algumas atividades reconhecidamente tradicionais e, entre elas, a de manter a vigilância dos pacientes em regime de internação e intervir nas situações que representam risco potencial ao paciente e à própria equipe. Desse modo, a carga psíquica é intensificada devido ao estado de alerta dos profissionais e a imprevisibilidade do quadro clínico dos pacientes, como relatado: 
É agitado, bem agitado! Tem momentos que tá calmo, mas tem horas que agita! [...] Porque que é agitado! $\mathrm{O}$ paciente tá bem, daqui a pouco do nada ele já levanta assim gritando, querendo agredir os outros pacientes e por conta disso agita os outros pacientes e o setor fica todo agitado. (TE 24)

Sempre de olho também, porque eles são imprevisíveis. A gente não relaxa nunca, porque de repente você pode perder um momento de atenção e ser surpreendida. (TE 28)

Saber que você precisa ficar atento a todo minuto porque não sabe qual a reação deles, você fica de um lado para o outro vigiando [...] tem uns que sempre precisa ter mais cuidados. (TE9)

Nessa situação de trabalho, devem-se considerar os efeitos negativos do exercício da vigilância sobre a saúde mental do trabalhador que são de considerável monta, pois tais efeitos implicam cargas psíquicas que se intensificam, principalmente na ausência de uma atividade motora, utilizada como estratégia para extravasar a energia pulsional acumulada. Na medida em que a energia se acumula, dá origem à ansiedade, à tensão e às doenças de origem psicossomática ${ }^{12}$.

Os agravos decorrentes das cargas psíquicas de trabalho são expressos no corpo do trabalhador, entre as quais se destacam as do ambiente hospitalar como as mais importantes, pois se somam às demais cargas laborais e, aparentemente, as potencializam sobremaneira. Especificamente, na área da enfermagem, o desgaste em consequência das tarefas é notório, desencadeando sobrecarga emocional, com sentimentos de angústia, estresse psicológico, síndromes depressivas, entre outros agravos, muitas vezes associados, também, a distúrbios físicos ${ }^{13}$.

Existe o reconhecimento do ambiente hospitalar como insalubre, penoso e perigoso para os trabalhadores, sendo um local de riscos, propício ao adoecimento e acidentes. Há, também, a alta pressão social e psicológica a que estão submetidos os profissionais de enfermagem, tanto na esfera laboral - devido às condições inadequadas de trabalho - quanto na qualidade de vida, o que podem acarretar ocorrências de transtornos mentais, como ansiedade e depressão ${ }^{8}$.

\section{Condições inadequadas de trabalho}

Evidenciou-se no estudo que, além das cargas psíquicas concernentes ao cuidado do paciente com transtorno mental, existem aquelas representadas pelas condições inadequadas de trabalho. A ausência de recursos materiais afeta o trabalho da equipe, principalmente diante de intercorrência clínica e necessidade de atendimento de pacientes que, além do transtorno mental, possuem comorbidades as quais exigem conhecimentos específicos e tecnologias do cuidado, sendo o desgaste acentuado, como referido pelo grupo:
Essa parte clínica que a gente não tem um suporte! Não tem um médico clínico 24 horas! Não tem material necessário para uma intercorrência mais séria! São todos esses problemas que a gente enfrenta aqui todos os dias. (E1)

Fico triste porque não tem material para atender o doente! Não só eu da enfermagem, mas os médicos e a equipe também reclamam. Não adianta pedir me dá isso me dá aquilo! Porque não vai ter. Não tem suporte para atender o paciente em caso de maior gravidade! Não tem material. (TE 19)

Aqui na enfermaria não veem o lado clínico do paciente! Então as nossas condições para trabalhar em qualquer intercorrência clínica são bem difíceis! Não tem recursos! Falta material e suporte para atuar numa parada. (TE4)

A precariedade das condições de trabalho contribui para a intensificação do trabalho, devendo-se atentar para os fatores de ordem estrutural e organizacional como o aumento da jornada, o acúmulo de funções e a maior exposição a fatores de risco para a saúde do trabalhador. Esses aspectos sinalizam o risco de aumento demasiado das cargas de trabalho entre esses profissionais e a necessidade de medidas que amenizem/eliminem essas cargas, como investimentos na melhoria das condições de trabalho desses indivíduos ${ }^{8}$.

A exposição do trabalhador de enfermagem aos riscos ocupacionais ocorre a partir de uma variedade de agentes e, entre eles, as cargas oriundas do trabalho como as biológicas, as psíquicas, as físicas e as ergonômicas. A vivência institucional, em algumas situações, é permeada por agravos à saúde como estresse, cansaço e distúrbios osteomusculares decorrentes de inadequações estruturais em termos de espaço físico, de equipamentos em quantidade e qualidade e de número insuficiente de profissionais de enfermagem, com necessidade de planejamento e ações para a promoção da saúde destes trabalhadores ${ }^{14}$.

Alerta-se que nem todas as instituições estão dispostas a investir em recursos para interromper ou minimizar o processo de exposição ocupacional e adoecimento dos profissionais, tampouco em ações que promovam a qualidade de vida no trabalho. Por isso, faz-se necessário impulsionar uma mudança de atitude em prol da vigilância da saúde no trabalho, tendo em vista os impactos na saúde do trabalhador, os encargos sociais e financeiros decorrentes de faltas, licenças médicas, afastamentos e comprometimento da qualidade do serviço prestado ${ }^{15}$.

\section{A reduzida autonomia frente à organização do trabalho}

Diante das cargas psíquicas e físicas decorrentes do cuidado ao paciente com transtorno mental e da precariedade das condições de trabalho, como a planta física inadequada e o déficit de recursos humanos, os trabalhadores de enfermagem, além de se sentirem 
sobrecarregados em sua função, destacam a reduzida autonomia no trabalho em seus depoimentos.

A enfermagem tem uma pouca autonomia aqui onde eu trabalho em relação aos outros profissionais envolvidos [...] são eles que tomam as atitudes. (TE7)

Não tem uma coordenação de enfermagem para garantir o seu lado hierárquico! [...] alguma eventualidade ou situação de trabalho com técnico é reportada direto para a direção! Muitas vezes, o funcionário não se sente bem! Não tem um coordenador de enfermagem. (TE15)

Não tem chefia direta de enfermagem! A gente tem que recorrer aos psicólogos que estão nas chefias e coordenações das enfermarias! A gente tem que ficar ligando para os psicólogos que não têm uma visão de enfermagem, para resolver as nossas questões. (E1)

Os trabalhadores de enfermagem da psiquiatria, apesar de realizarem um trabalho relevante no contexto do cuidado em saúde mental, nem sempre se sentem reconhecidos pelos demais integrantes da equipe multiprofissional. A reduzida participação nas decisões, no que diz respeito ao tratamento, acarreta insatisfação no grupo, principalmente ao se considerar que são trabalhadores que, por prestarem cuidados contínuos aos pacientes, possuem saberes práticos, essenciais, mas nem sempre considerados pela organização ${ }^{6}$.

Para tanto, há necessidade de reflexão no âmbito da subjetividade desses profissionais, a sua implicação com os serviços e a perspectiva que têm do próprio trabalho, que influenciam diretamente no seu comprometimento com as práticas profissionais. Somados a isso estão, ainda, outros fatores, como a exposição às dificuldades dos usuários, condições e qualidade de vida, apatia e ausência de autonomia com relação à prática e aos recursos, além do envolvimento com o outro, o que influencia diretamente na satisfação profissional da equipe dos serviços de saúde mental ${ }^{4}$.

\section{Implicações das cargas de trabalho para a saúde: o sofrimento no trabalho}

$\mathrm{O}$ estresse psicológico no campo de estudo foi a principal queixa referida pelos trabalhadores de enfermagem, o qual perpassou pelas implicações das cargas de trabalho para a saúde mental do grupo. Acrescentam-se outras queixas de ordem subjetiva como, incômodo, nervosismo, irritação, tensão, desgaste, ansiedade, impaciência e angústia - como relatadas a seguir:

Fico estressada, às vezes! Tem dia que não consigo nem ouvir uma música devido ao barulho da enfermaria! Fazendo esforço além do necessário durante a contenção física ou separando brigas. (TE 18)

[...] porque na minha fragilidade fui me deixando... ficando estressada mas sem querer passar para os meus pacientes e também até para os meus supervisores, mas eu comecei a sofrer de pressão no trabalho. (TE22)
Estressante. É estressante sim, eu não fico nervosa, irritada à toa... mas eu fico triste! Logo na primeira semana que comecei a trabalhar aqui eu queria sair correndo! Chorei. (E3)

Frequentemente, na literatura, os profissionais de enfermagem da psiquiatria são referidos como uma categoria particularmente exposta ao estresse ocupacional, em consequência das contínuas demandas físicas e emocionais requeridas pelos pacientes. Acrescenta-se que o trabalhador, geralmente, possui mais de um vínculo empregatício, em sua maioria pertence ao gênero feminino, configurando a tripla jornada de trabalho, devido às atividades domésticas, com pouco tempo destinado ao lazer, à prática de exercícios e ao cuidado com a própria saúde ${ }^{6}$.

O sofrimento mental dos trabalhadores da saúde é capaz de acarretar morbidades diversas, com determinações estruturais, haja vista que as instituições de saúde vivenciam condições inadequadas de trabalho com baixa resolutividade dos serviços, acarretando sobrecarga de trabalho e estresse. Além disso, aludem-se às características do trabalho em saúde, que envolve lidar cotidianamente com a dor, a carência econômica e a doença dos usuários, situações que afetam, emocionalmente, os profissionais ${ }^{16}$.

\section{Conclusões}

Evidenciou-se, no estudo, que os trabalhadores de enfermagem da psiquiatria encontram-se expostos às excessivas cargas físicas e psíquicas do trabalho, por conviverem diuturnamente com o sofrimento psíquico de usuários e familiares e desenvolverem suas atividades laborais em condições inadequadas. $\mathrm{Na}$ origem das cargas, devem ser consideradas, também, outras exigências de cunho técnico que demandam esforços físicos acentuados, em decorrência do cuidado de pacientes com dependência parcial e/ou total da enfermagem por se encontrarem acamados, sedados e/ou contidos. Além disso, contribuem para os resultados a falta de autonomia profissional e a organização do trabalho.

Outras evidências contribuem para a excessiva carga no trabalho de enfermagem em hospital psiquiátrico, como a precariedade das condições laborais em que se constatou a insuficiência de recursos humanos e materiais, sobrecarregando as equipes devido ao volume de trabalho a ser executado. Quanto à organização, os trabalhadores referiram a limitada autonomia no trabalho e lastimaram o fato de não terem uma chefia de enfermagem que os representassem e também por não se sentirem apoiados pela coordenação, razão de insatisfação e desmotivação no trabalho.

Devido aos fatores de ordem organizacional, estrutural e relacional, as cargas de trabalho são intensificadas levando ao sofrimento no trabalho e implicações para a saúde do trabalhador diante de queixas como: 
estresse, cansaço físico e mental, dores no corpo, ansiedade e fadiga. Cabe ressaltar que o estresse psicológico foi a principal queixa referida pelos trabalhadores diante das cargas em hospital psiquiátrico, o que pode levar ao adoecimento no trabalho, caso a organização não adote medidas preventivas desses desgastes.

Apesar das limitações do estudo relativas ao número reduzido de participantes e por ter sido realizado em uma única unidade de internação, foram apontadas questões relevantes acerca das cargas decorrentes do trabalho em hospital psiquiátrico e suas repercussões para a saúde do trabalhador de enfermagem. Sugere-se a continuidade de pesquisas desta natureza, assim como a criação de um espaço dedicado à discussão e ao enfrentamento dos problemas referidos pelos trabalhadores do serviço, considerando que o campo do estudo não possuía um serviço de saúde do trabalhador, na ocasião em que os dados foram coletados.

\section{REFERÊNCIAS}

1.Mininel VA, Baptista PCP, Felli VEA. Cargas psíquicas e processos de desgaste em trabalhadores de enfermagem de hospitais universitários brasileiros. Rev Latino-Am Enfermagem [periódico na internet] 2011 [citado em 17 jul 2015] 19:1290-7. Disponível em: http://www. scielo.br/scielo.php?script $=$ sci_abstract $\&$ pid $=$ S0104 $11692011000200016 \& \operatorname{lng}=\mathrm{pt} \& \bar{n} r \mathrm{rm}=\mathrm{iso} \& \operatorname{lng}=\mathrm{pt}$

2.Jorge MSB, Pinto DM, Quinderé PHD, Pinto AGA, Souza FSP, Cavalcante CM. Promoção da saúde mental tecnologias do cuidado: vínculo, acolhimento, co-responsabilização e autonomia. Ciênc saúde coletiva [periódico na internet] 2011 [citado em mai 2015] 16:3051-60. Disponível em: http://www.scielo.br/scielo.php?pid=S1413$81232011000800005 \&$ script $=$ sci_arttext

3.Moreira LHO, Felipe ICV, Goldstein EA, Brito AP, Costa LMG. A inclusão social do doente mental: contribuições para a enfermagem psiquiátrica. Inclusão Social [periódico na internet] 2008 [citado em 17 jun 2015] 3:35-42. Disponível em: http://revista.ibict.br/inclusao/index.php/ inclusao/article/view/104.

4. De Marco PF, Cítero VA, Moraes E, Nogueira-Martins LA. O impacto do trabalho em saúde mental: transtornos psiquiátricos menores, qualidade de vida e satisfação profissional. J Bras Psiquiatr. [periódico na internet] 2008 [citado em 15 ago 2015] 57:178-83. Disponível em: http://www. scielo.br/pdf/jbpsiq/v57n3/04.pdf.

5.Magnus CN, Merlo ARC. Sofrimento-prazer: dinâmica do trabalho de profissionais de saúde mental de um hospital psiquiátrico público. PSICO [periódico na internet] 2012 [citado em 15 ago 2015] 43:185-92 Disponível em: http:// revistaseletronicas.pucrs.br/ojs/index.php/revistapsico/ article/view/11695

6.Carvalho MB, Felli VEA. O trabalho de enfermagem psiquiátrica e os problemas de saúde dos trabalhadores. Rev Latino-Am Enfermagem [periódico na internet] 2006 [citado em 15 ago 2015] 14: 61-9. Disponível em: http: http:// www.scielo.br/scielo.php?script $=$ sci_arttext\&pid=S0104 $11692006000100009 \& \operatorname{lng}=\mathrm{pt} \& \mathbf{E} \mathbf{m}=$ iso

7.Selligmann-Silva E. Trabalho e desgaste mental: o direito de ser dono de si mesmo. São Paulo: Cortez; 2011.

8.Schmoeller R, Trindade LL, Neis MB, Gelbckel FL, Pires DEP. Cargas de trabalho e condições de trabalho da enfermagem: revisão integrativa. Rev Gaúcha Enferm. [periódico na internet] 2011 [citado em 15 ago 2015] 2:368-77. Disponível em: http://dx.doi.org/10.1590/S1983. 14472011000200022.

9.Minayo, MCS. O desafio do conhecimento: pesquisa qualitativa em saúde. 13ª ed. São Paulo: Hucitec; 2013.

10.Bardin L. Análise de conteúdo. Lisboa(Pt): Edições 70; 2011.

11. Mello MT. Trabalhador em turno: fadiga. Belo Horizonte(MG): Editora Atheneu; 2013.

12.Dejours C. Psicodinâmica do trabalho: contribuições da escola dejouriana à análise da relação prazer, sofrimento trabalho. São Paulo: Atlas; 2012.

13.Secco IAO, Robazzi MLCC, Souza FEAS, Shimizu DS. Cargas psíquicas de trabalho e desgaste dos trabalhadores de enfermagem de hospital de ensino do Paraná, Brasil. SMAD, Rev Eletrônica Saúde Mental Álcool Droga. [periódico na internet] 2010 [citado em 15 ago 2015] 57:1-17. Disponível em: http://pepsic.bvsalud.org/scielo.php?script=sci_arttext \&pid=S1806-69762010000100016

14.Souza NVDO, Pires AS, Gonçalves FGA, Cunha LS, Shoji S, Ribeiro LV, Tavares FA. Riscos ocupacionais relacionados ao trabalho de uma unidade ambulatorial especializada. Rev enferm UERJ. [periódico na internet] 2012 [citado em 15 ago 2015] 20:609-14. Disponível em: http://www.facenf.uerj.br/v20nesp1/v20e1a10.pdf 15.Mininel VA, Felli VEA, Silva EJ, Torri Z, Abreu AP, Branco MTA. Cargas de trabalho, processos de desgaste e absenteísmo doença na enfermagem. Rev Latino-Am. Enfermagem [periódico na internet] 2013 [citado em 10 jul 2015] 21:1290-7. Disponível em: php?script=sci abstract\&pid =S0104-11692013000601290\&lng=pt\&nr $\mathrm{m}=$ iso\&tlng $=\mathrm{pt}$

16.Sarquis LMM, Felli VEA. Os sentimentos vivenciados após exposição ocupacional entre trabalhadores de saúde: fulcro para repensar o trabalho em instituições de saúde. Rev Bras Enferm. [periódico na internet] 2009 [citado em 15 jun 2015] 62:701-4. Disponível em: http://www. scielo.br/scielo.php?script=sci_arttext\&pid=S0034$71672009000500008 \& \operatorname{lng}=\mathrm{pt} \& \overline{\mathrm{n} r m}=\mathrm{iso}$ 